\title{
Active Disturbance Rejection Control for Activated Sludge Wastewater Treatment Processes
}

\author{
Li Meng1, a, Xuelian Zhang 2,b, Lin Qiao, c, and Xiaowei Han 1, d \\ 1 Department of Information Engineering, Shenyang University, Shenyang, 110044, China \\ 2 Shenshuiwan Sewage Treatment Plant of Guodian Northeast Environmental Protection Industry \\ Group Co., Ltd., Shenyang, 110141, China \\ a mengliinsy@126.com, b105438758@qq.com, c1031904597@qq.com, dhxw69@163.com
}

Keywords: Activated Sludge Process, Active Disturbance Rejection Control (ADRC), Tracking Differentiator (TD), Extended State Observer (ESO), Dissolved Oxygen (DO)

Abstract. In this article, Active Disturbance Rejection Control (ADRC) is proposed for the control of the activated sludge wastewater treatment processes. Simulation results show that the dissolved oxygen concentration can track the set value with non-overshoot and short settling time as possible by ADRC. Under disturbance, ADRC can actively estimate and compensate for external disturbances, to ensure a good control effect of dissolved oxygen concentration.

\section{Introduction}

Activated Sludge Process (ASP) is one of the widely used sewage treatment methods. It consists of two parts: reaction cell (aeration tank) and sedimentation tank (shown in Fig. 1).

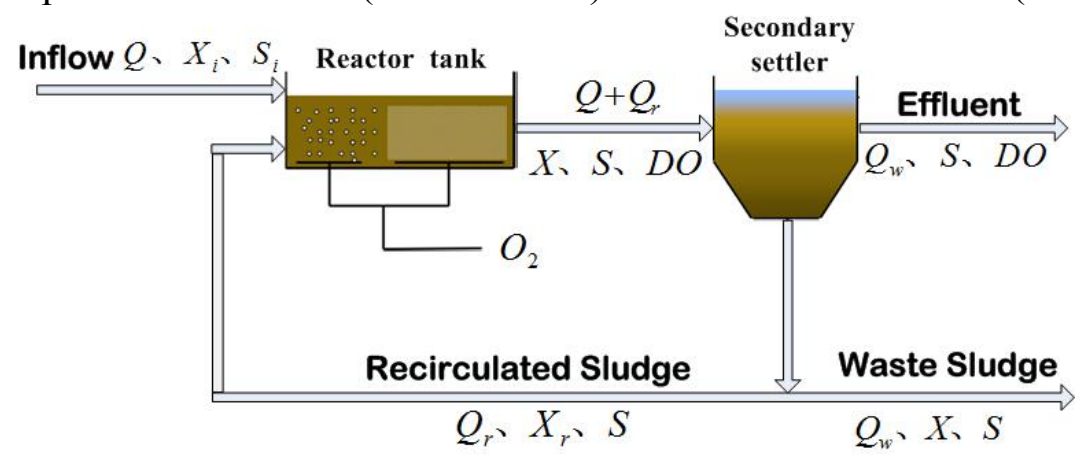

Fig.1. Simple configuration for the activated sludge process

Biochemical reactions in the reaction tank play a vital role in the purification of sewage. The biochemical reaction process in the reaction tank requires the participation of dissolved oxygen (DO), thus the dissolved oxygen concentration will determine the degree of biochemical reactions in the sewage treatment process $[1,2]$. Whether from the sewage treatment process itself, or from the energy saving, energy consumption point of view, the effective control of dissolved oxygen is very important.

A variety of control methods were used for dissolved oxygen control. Traditional PI / PID control [3-5] in the dissolved oxygen control has achieved some success. However, the effluent quality is affected by many influencing factors, such as influent composition, flow rate, sludge concentration, dissolved oxygen content, and so on. It is a typical nonlinear and strong coupling process [1]. But PI / PID cannot actively resist the effect of disturbance on the control and the control effect on the nonlinear and the strong coupling process is limited. And the control effect of the controlled process is implemented only after the deviation is generated. Aiming at the particularity and complexity of wastewater treatment process control, various advanced control and intelligent control strategies have been proposed. Based on the model of Activated Sludge Model No. 1 (ASMl), a fuzzy model is established to design a predictive control to keep the dissolved oxygen concentration constant in the reactor [6]. Based on the self-organizing neural network model, self-organizing neural network Model Predictive Control is designed to maintain concentration of dissolved oxygen a constant in the wastewater treatment system [7]. The modeling control method based on the feed-forward neural 
network[8] and dissolved oxygen optimization control method based on fuzzy control [9] have been reported in the dissolved oxygen control research.

In fact, the sewage influent component, the influent flow, the sludge concentration and so on are the disturbance factor to influence water quality. The traditional sewage treatment control method is difficult to solve this problem which the sewage treatment process must solve. However, treating various factors affecting the dissolved oxygen concentration as disturbances, the dissolved oxygen control problem is considered as the active disturbance rejection control (ADRC) [10]. The essence of ADRC is disturbance estimation and compensation by the Extended State Observer (ESO). Nonlinear mechanism is introduced to further improve the control performance. In this paper, nonlinear ADRC strategy is proposed to keep the dissolved oxygen value constant and to purify the wastewater.

\section{Mathematic Model Based on the Sewage Treatment Process of Activated Sludge}

In order to develop the respective mathematical model, the following facts are assumed [11 -13]:

The specific growth rate of microorganisms is bigger that their death rate. The specific growth rate is considered to obey the Monod law, which describes the specific growth rate as a function only of substrate and oxygen concentration, independently of other nutrients concentration, and other environmental factors.

- No biochemical reactions take place inside the settling tank.

- Biomass in the sedimentation tank is negligible.

- The inflow stream contains no biomass.

- Complete settling is achieved, hence the sludge wastage is restricted to waste stream.

- The recycling of activated sludge affects the sludge age and the yield coefficient.

By means of mass balance for biomass concentration, substrate concentration and oxygen concentration, we obtain the following model [11]

$$
\left\{\begin{array}{c}
\frac{d X}{d t}=\frac{2 S \cdot X}{(46+S)} \frac{D O}{(2+D O)}-0.37 X+600 \\
\frac{d S}{d t}=-\frac{3.33 S \cdot X}{(46+S)} \frac{D O}{(2+D O)}+600-4 S \\
\frac{d D O}{d t}=-2.14 \frac{S \cdot X}{(46+S)} \frac{D O}{(2+D O)}-0.071 X+u
\end{array}\right.
$$

The control problem can be described as the design of control law $u$, which makes the dissolved oxygen concentration DO within the ideal range (usually within the $2-4 \mathrm{~g} / \mathrm{m}^{3}$ ).

\section{Active Disturbance Rejection Control (ADRC)}

The basic idea of ADRC is to transfer a nonlinear system to a linear system by estimating and compensating the external disturbances and internal uncertainties in real time, which is also called linearization with dynamic compensation in [14]. For a coupled system, the decoupling control can be realized by treating the coupled parts as model uncertainties. A classical Active Disturbance Rejection Controller (ADRC) mainly consists of Tracking Differentiator (TD), Extended State Observer (ESO) and Nonlinear State Error Feedback (NLSEF) control law. An ADRC for an Nth order system is shown in Fig. 2. 


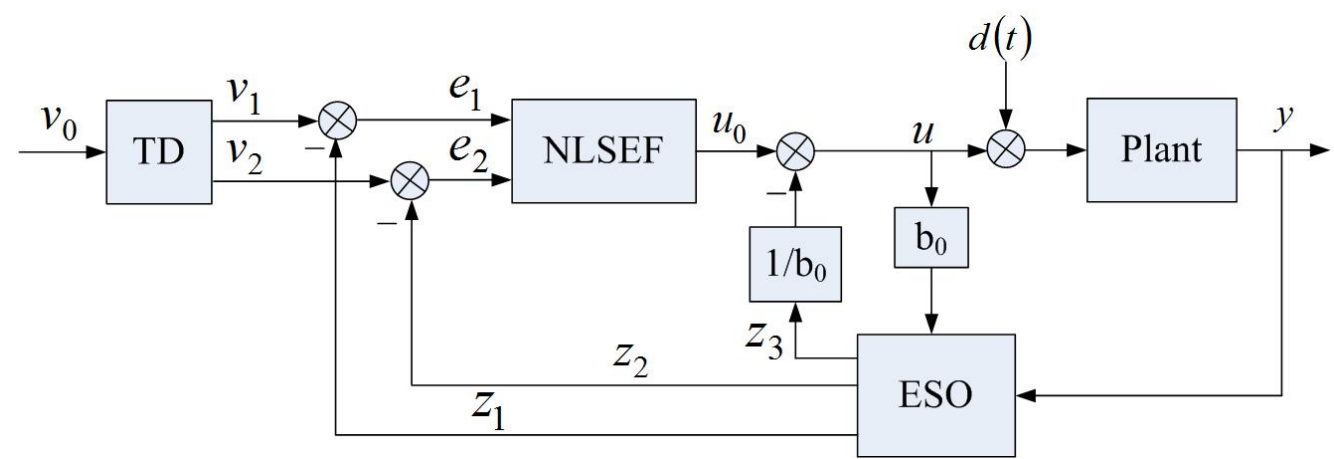

Fig. 2. Block diagram of ADRC

Specifically, the TD is capable of tracking the input signal and providing its high-quality differential signals without amplification of noises. The tracking signal can smooth the sudden changes in the input signal, and results in an ease of contradiction between overshoot and fast response requirement. The ESO is the core of ADRC. It can observe state variables, disturbances and uncertainties of the system, depended only on system inputs and outputs. The NLSEF is responsible for dealing with errors between the outputs of the TD and the ESO [14].

For a two order system, based on the maximum convergence rate control function fhan, the discrete tracking differentiator is proposed [15]

$$
\left\{\begin{array}{c}
x_{1}(k+1)=x_{1}(k)+h x_{2}(k) \\
x_{2}(k+1)=x_{2}(k)+h \cdot f h \\
f h=\text { fhan }\left(x_{1}(k)-v_{0}(k), x_{2}(k), r, h\right)
\end{array}, \text { where fhan }\left(x_{1}, x_{2}, r, h\right)=-\left\{\begin{array}{cc}
r \operatorname{sign}(a), & |a|>d \\
r \frac{a}{d}, & |a| \leq d
\end{array}\right.\right.
$$

where $h$ is the step size, $r$, called the speed factor, which make the system reach the steady state with the fastest speed without oscillation.

Nonlinear state error feedback is

$$
\begin{aligned}
& u=\beta_{1} f a l\left(e_{1}, \alpha_{1}, \delta\right)+\beta_{2} \operatorname{fal}\left(e_{2}, \alpha_{2}, \delta\right), \quad 0<\alpha_{1}<1<\alpha_{2} \\
& \text { where } \operatorname{fal}(x, \alpha, \delta)=\left\{\begin{array}{cc}
\frac{x}{\delta^{1-\alpha}}, & |x|<\delta \\
|x|^{\alpha} \operatorname{sign}(x), & |x| \geq \delta
\end{array}\right.
\end{aligned}
$$

Generally in ADRC, $\alpha$ is chosen as $0.25,0.5$ or 1.5 . The big difference between nonlinear state error feedback and PID error feedback is the total elimination of the integral element, thus avoiding the integral saturation, unstable oscillation and slow system.

ESO is the improvement of the state observer, which can estimate the disturbance in real time, and is the core part of the active disturbance rejection controller. Frequently-used nonlinear form is

$$
\left\{\begin{array}{c}
e=z_{1}-y, f e=f a l(e, 0.5, \delta), f e_{1}=f a l(e, 0.25, \delta) \\
z_{1}(k+1)=z_{1}(k)+h\left(z_{2}-\beta_{01} e\right) \\
z_{2}(k+1)=z_{2}(k)+h\left(z_{3}-\beta_{02} f e+b_{0} u\right) \\
z_{3}(k+1)=z_{3}(k)+h\left(-\beta_{03} f e_{1}\right)
\end{array}\right.
$$

ESO is generally one order higher than the controlled object. For the n-order system, the $n+1$ order $\mathrm{ESO}$ is based on the $\mathrm{N}$ state variables and an observation variable. 
ESO, only an estimate of the disturbance, is combined with the disturbance compensation to realize capacity of resisting disturbance. The state error feedback control $u_{0}$ with the compensation of the disturbance estimation $z_{3}$ is the final $\operatorname{control}_{u}=u_{0}-z_{3} / b_{0}$.

\section{Designs and Simulation Analysis of the ADRC of Dissolved Oxygen Concentration}

Based on the sewage treatment process of activated sludge, ADRC control of the dissolved oxygen concentration is studied.

1) Tracking of the set value of dissolved oxygen concentration

For the dissolved oxygen concentration set value $2 \mathrm{~g} / \mathrm{m}^{3}$, ADRC control parameters are chosen as followed.

TD: $h=0.0025, r=3200, h_{0}=0.0125$.

ESO: $\beta_{01}=400, \beta_{02}=5000, \beta_{03}=61664, b=0.38$.

NLSEF: $\beta_{1}=80, \beta_{2}=0.00001$.

In Fig.3, the comparison between PID controller and ADRC for tracking of the set value is shown. ADRC make the dissolved oxygen concentration achieve satisfactory both overshoot and settling time.

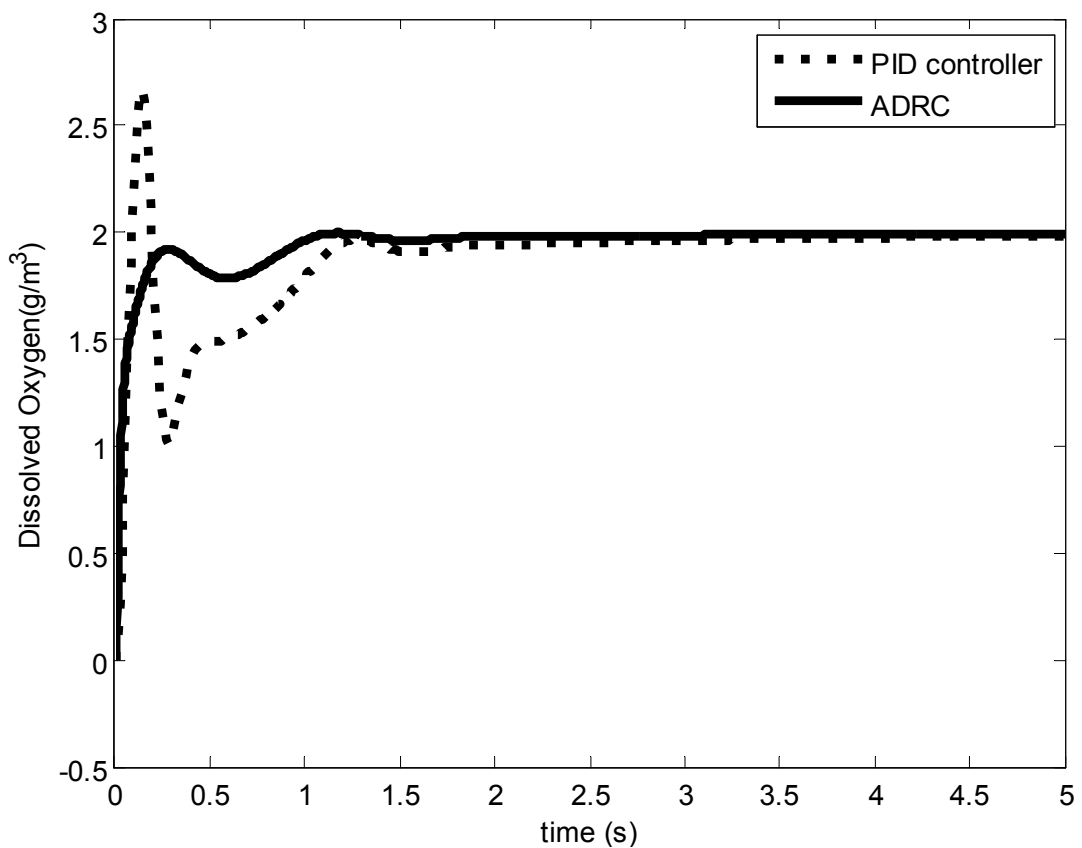

Fig. 3. Comparison of PID controller and ADRC for tracking of the set value

2) Tracking of the set value of dissolved oxygen concentration with Step disturbance and Sinusoidal disturbance

With a step disturbance added at $15 \mathrm{~s}$ and keeping the control parameters unchanged, the dynamic response of PID control system and ADRC control system are shown in Fig.4.

In Fig.4, Dissolved Oxygen in ADRC system possesses smaller vibration, caused by step disturbance, than those in PID controlled system.

With a sinusoidal disturbance $\mathrm{d}(\mathrm{t})=10 \sin (20 \pi \mathrm{t})$ and keeping the control parameters unchanged, the dynamic response of PID control system and ADRC control system are shown in Fig.5 and Fig.6.

It can be seen from Fig. 5 and Fig. 6 that, when there is sinusoidal disturbance, ADRC can still obtain better control effect of dissolved oxygen concentration. This indicates that the ADRC can actively estimate and compensate for external disturbances, to ensure that the system has a good control effect of dissolved oxygen concentration. 
with step disturbance

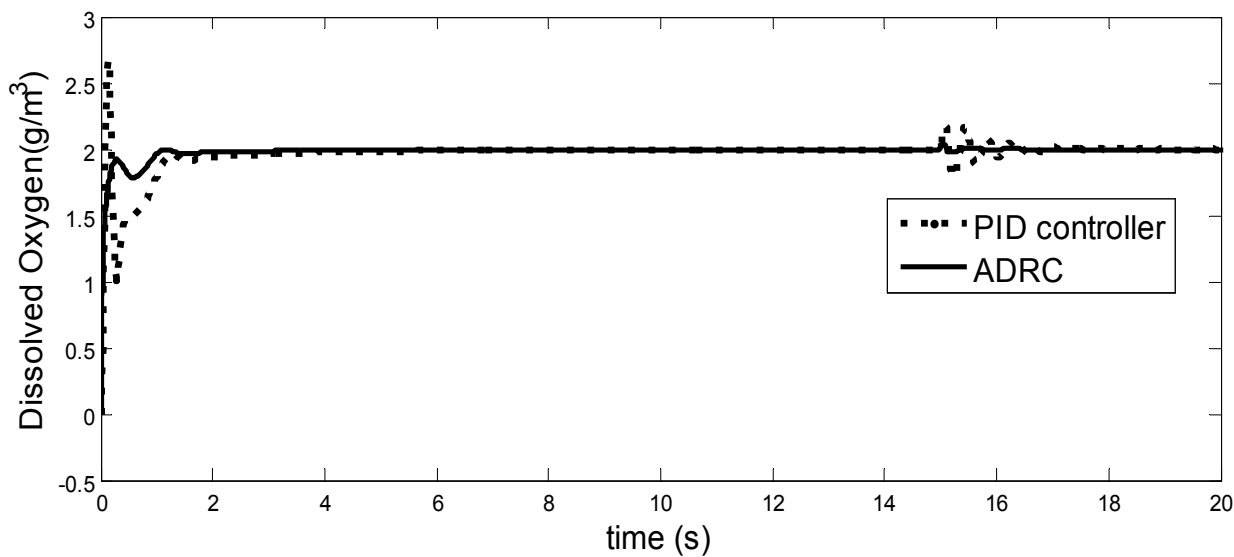

Fig. 4 Comparison of PID controller and ADRC for tracking of the set value with Step disturbance

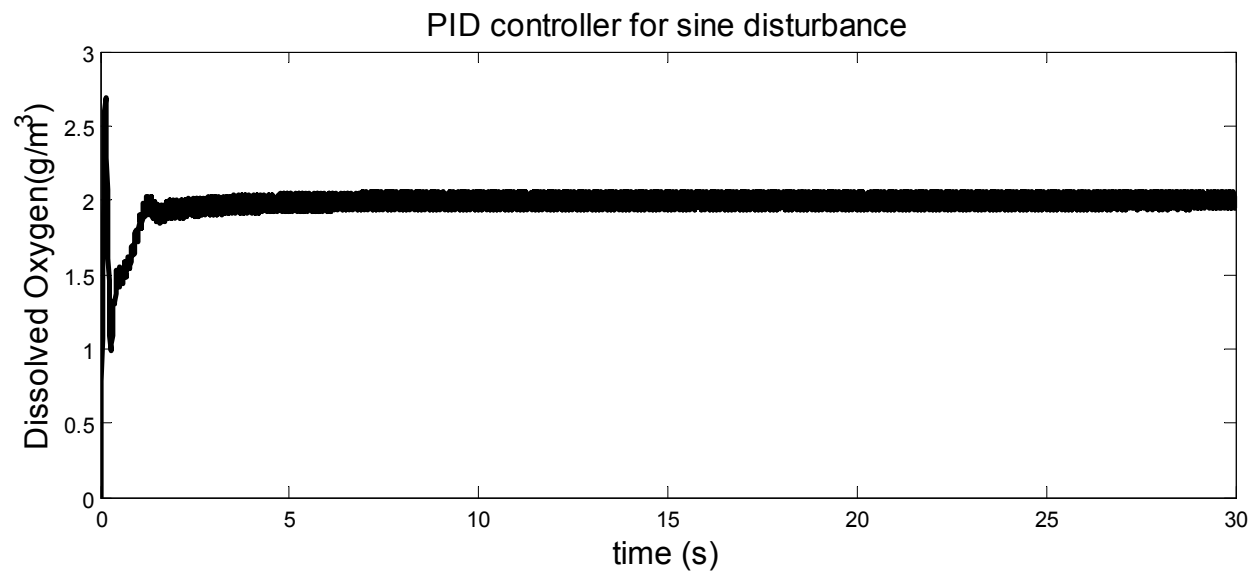

Fig. 5 PID controller for tracking of the set value with Sinusoidal disturbance

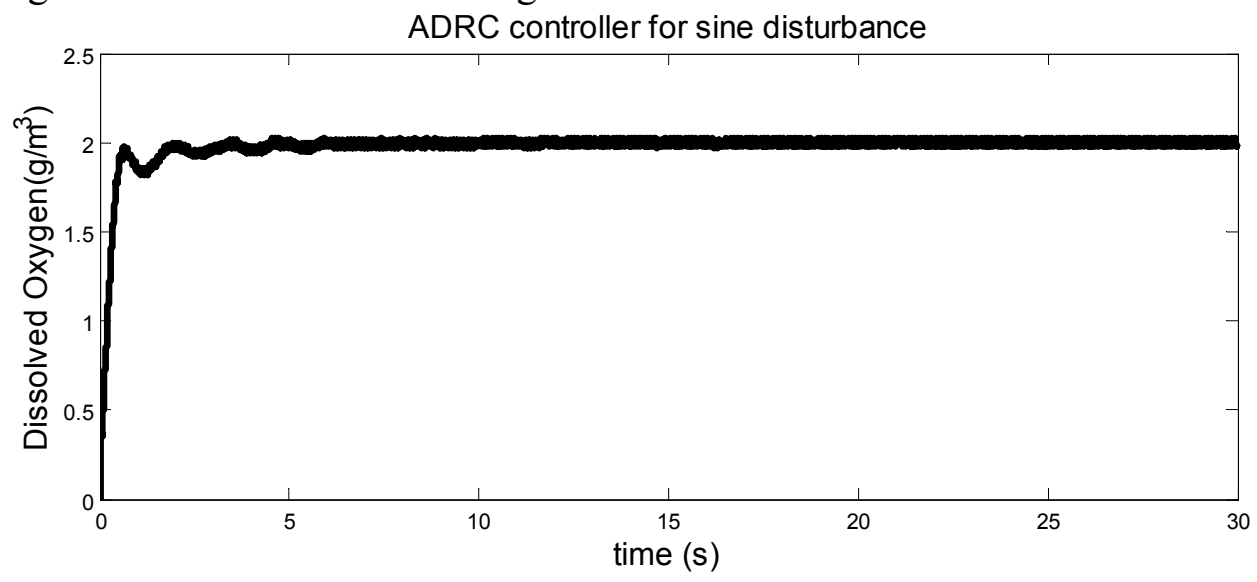

Fig.6 ADRC for tracking of the set value with Sinusoidal disturbance

\section{Conclusions}

In this paper, a nonlinear auto-disturbance rejection controller is designed for the control of the dissolved oxygen concentration in the wastewater treatment process. The simulation results show that, despite various set value and external disturbance, the disturbance can be effectively estimated and compensated by the ADRC to obtain the good control effect of the dissolved oxygen concentration in order to promote the biochemical reaction in the sewage treatment process. 


\section{Acknowledgements}

This work was financially supported by Liaoning Building Project of Public Welfare Research Team in Science (GY2015-E-009) and Shenyang Program for Tackling Key Problems in Science and Technology (F14-129-9-00).

\section{References}

[1]B Holenda., E Domokos, A Redey, J Fazakas. Dissoloved oxygen control of the activated sludge wastewater treatment process using model predictive control[J]. Computer and Chemical Engineering, 2008, 32(6): 1270-1278.

[2]A C B Carlos, A M A Rui, A C L Jorge. Dissolved oxygen control of the activated sludge wastewater treatment process using stable adaptive fuzzy control[J]. Computers and Chemical Engineering, 2012, 37(10): 152-162.

[3]B Carlsson, C F Lindberg, S Hasselblad, S Xu. Online estimation of the respiration rate and the oxygen transfer rate at Kungsangen wastewater plant in Uppsala $[\mathrm{J}]$. Water Science and Technology, 1994, 30(4): 255-263.

[4]B Carlsson, A Rehnstrem. Control of an activated sludge process with nitrogen removal - a benchmark study[J]. Water Science and Technology, 2002, 45(4-5): 135-142.

[5]L Amand, B Carlsson. Optimal aeration control in a nitrifying activated sludge process[J].Water Research, 2012, 46(7): 2101-2110.

[6]Yang Ting, Qiu Wei, Ma You, Mohammed Chadli, Zhang Lixian. Fuzzy model-based predictive control of dissolved oxygen in activated sludge processes[J]. Neurocomputing, 2014, 136(20): 88-95.

[7]Han Hong-Gui, Qiao Jun-Fei, Chen Qi-Li. Model predictive control of dissolved oxygen concentration based on a self-organizing RBF neural network[J]. Control Engineering Practice, 2012, 20(4): $465-476$.

[8] HAN Guang, QIAO Jun-fei, BO Ying-chun. Feedforward neural network modeling and control for dissolved oxygen concentration [J]. Control Theory \& Applications, 2013, 30(5): 585-591.

[9] Liu Zaiwen , Xu Jiping , Yang Bin , Hou Chaozhen and Cheng Zhiqiang. The method Of DO optimal control for SBR wastewater treatment process [J]. Computers and Applied Chemist, 2007, 24(2): 231-234.

[10] Han J. From PID to active disturbance rejection control [J]. IEEE transactions on Industrial Electronics, 2009, 56(3): 900-906.

[11] Wei Wei, Wang Meng, Liu Zaiwen, Wang Xiaoyi. Linear Active Disturbance Rejection Control for Activated Sludge Wastewater Treatment Processes [J], Computer Simulation, 2015,32(8): 417-421.

[12]N S Edgar, M G Jose, R Esperanza. Minimal PD fuzzy control of a wastewater treatment plant[C]. Proceedings of the 15 IEEE International Symposium on Intelligent Control, Greece, 2000: 169-173.

[13]D Orhon, N Artan. Modelling of Activated Sludge System [M]. USA: Technomie Publisher Co., Lancaster, PA, 1994.

[14] J. Han, "From PID to active disturbance rejection control," IEEE Trans. Ind. Electron., vol. 56, no. 3, pp. 900-906, Mar. 2009. 